\title{
Impact of Activation Energy in Darcy-Forchheimer Flow of Cross Nanofluid over a Radial Stretching Surface with Viscous Dissipation and Joule Heating
}

\author{
Cherlacola Srinivas Reddy ${ }^{1}$, Besthapu Prabhakar ${ }^{2 *}$ \\ ${ }^{1}$ Department of Mathematics, ABV Government College, Janagaon 506167, Telangana, India \\ ${ }^{2}$ Department of Mathematics, Kakatiya Government College, Hanamkonda 506001, Telangana, India
}

Corresponding Author Email: prabhakarbesthapu@gmail.com

https://doi.org/10.18280/ijht.390518

Received: 1 June 2020

Accepted: 17 September 2021

\section{Keywords:}

Darcy-Forchheimer flow, cross fluid, joule heating, viscous dissipation, radially stretching surface

\begin{abstract}
This framework analyzes the impact of activation energy (AE) and binary chemical reaction (BCR) in Darcy-Forchheimer flow of cross fluid with nanoparticles due to radially stretched surface. Moreover slip, joule heating and viscous dissipation aspects have been considered. Ordinary differential equations acquired from the modelled governing partial differential equations with the assistance of suitable transformations. Further the system of nonlinear equations is computed numerically by Runge-Kutta-Fehlberg method cum shooting technique. Graphical representation has been given to analyze the velocity, temperature and concentration fields with the effect of various pertinent parameters. It is evident that inertia coefficient declines the velocity. Velocity decays for larger Weissenberg number while opposite trend observed in temperature field. Temperature field rises for augmented values of Eckert number. Concentration increases with increase of energy parameter.
\end{abstract}

\section{INTRODUCTION}

The study of non-Newtonian liquids flow and their features grab the attention of many researchers and scientists due to their significant applications in industrial products and procedures. Non-Newtonian liquids possess the non-linear relation between stress and strain. Depends on high viscosity few commonly used non-Newtonian fluids are toothpaste, lubricants, ketchup, silly putty, syrup, honey, paint, plastic, etc. Investigation of non-Newtonian flow field and its characteristics is quite difficult as compared to Newtonian liquids. To describe the features of non-Newtonian fluid flow by the well known Navier-Stokes equations are inadequate. Therefore, various fluid models have been suggested to demonstrate the features of non-Newtonian fluids. Cross [1] proposed Cross fluid model which is a major subclass of generalized Newtonian fluids and it predicts the shear thinning effects for both high and low shear rates. This fluid model has remarkable applications in engineering calculations due to the existence of the time constant. The empirical study of the cross fluid model was presented by Escudier et al. [2] by considering Cross equation with the non-Newtonian liquids carboxy methyl cellulose (CMC), xanthan gum (XG) and illustrated the fluid flow data. Xie and Jin [3] analyzed the free surface flow of the non-Newtonian fluid by WC-MPS method to find the four rheology parameters of Cross fluid model. Khan et al. [4] considered Cross fluid flow and heat transfer over a linear stretching surface and employed a numerical technique to solve boundary layer equations of the problem. From this study it is noticed that velocity profile is decreased with enlarging the Weissenberg number whereas inverse trend identified for the temperature field. Fluid flows through porous media have numerous applications corresponding to chemical engineering and geophysical systems. Such applications may include movement of water in reservoirs fermentation process, crude oil production, grain storage, ground water systems, ground water pollution and recovery systems etc. The Darcy's law is reasonable under circumstances of low velocity and little porosity but it is inadequate whenever inertial and boundary features occur at higher velocity. Under such conditions it is impossible to neglect the inertia and boundary impacts. The non-Darcian porous medium is the revised form of Darcy law which includes the inertia and boundary effects. For higher velocity flow, the Forchheimer [5] formula is used in which squared velocity term has been added in the equation to predict the behavior of inertia and boundary effects. Muskat [6] termed this as "Forchheimer factor" which always reasonable for large Reynolds number. Darcy-Forchheimer flow of non-newtonian fluid was analyzed by Seddeek [7]. Pal and Mondal [8] studied influence of the Lorentz forces in a non-Darcy flow by considering variable viscosity. Shehzad et al. [9] considered Cattaneo-Christov heat flux model and homogeneous-heterogeneous reactions in Darcy-Forchheimer flow of Oldroyd-B fluid. Their analysis reveals that temperature is diminishing for Cattaneo-Christov heat flux model when compared to classical Fourier's law of heat conduction. Hayat et al. [10] examined Cattaneo-Christov model in flow of Maxwell fluid through a non-Darcy porous medium with temperature-dependent thermal conductivity. Fluids cooling and heating is the major issue in many industrial fields such as power manufacturing and transportation. Efficient cooling techniques are required to cool any kind of high energy equipment. The working fluids which are utilized in the industries have poor thermal conductivity due to this they cannot meet modern cooling challenges. Therefore, to improve the heat transfer capabilities of common heat transfer fluids Choi and Eastman [11] proposed the idea of nanofluids which is mixture of tiny 
metallic particles in the base fluids. Nanoliquids boost the thermal performance of the base fluids. Masuda et al. [12] noticed that thermal conductivity improvement by mixing the nanoparticles in the fluid. Buongiorno [13] established seven slip mechanisms in which he identified that Brownian diffusion and thermophoresis are two major influential factors which enhances thermal conductivity of the fluids. First time Khan and Pop [14] studied nanofluid flow over a stretched surface and obtained numerical solutions through Keller-box method. Makinde and Aziz [15] used convective boundary condition instead of constant heat flux and examined the boundary layer flow of nanofluid induced by a stretching sheet. A revised model is proposed by Kuznetsov and Nield [16] in which nanoparticles at the surface are passively controlled. Analysis of various thermo physical aspects of few non-Newtonian nanofluids given in the studies [17-20]. The consideration of mass transport with activation energy is attracted the investigators due to its vast usage in prominent areas like geothermal engineering, chemical engineering, oil emulsions and food processing. Activation energy is suggested by Arrhenius in 1889. It is the minimum required energy to be acquired by particles to experience a chemical reaction and this can be existing in the form of kinetic energy or potential energy and without the activation energy, reactants cannot produce products. Once the reaction starts activation energy of the system becomes zero. Generally, activation energy (AE) is denoted by $E_{a}$ and writen in $\mathrm{Kj} / \mathrm{mol}$ or $\mathrm{Kcal} / \mathrm{mol}$. The applications of $\mathrm{AE}$ are very wide in geothermal engineering, chemical engineering, oil emulsions and food processing. Firstly Bestman [21] analyzed convective flow of binary amalgam in porous medium. $\mathrm{AE}$ and $n^{\text {th }}$ order chemical reaction effects on time dependent radiated flat porous plate is investigated by Makinde et al. [22]. Alsaadi et al. [23] studied nonlinear mixed convective flow of non-Newtonian nanoliquid over a porous stretching sheet under the impact of nonlinear radiation and activation energy and also, they discussed entropy generation rate. In this study they concluded that concentration increased with the effect of activation energy parameter. Unsteady flow of Carreau nanofluid is established by Irfan et al. [24] to acquire the effects of binary chemical reaction and activation energy. They reported the variations in both shear thinning and shear thickening fluids with the impact of reaction rate parameter and it shows the declined the concentration whereas it heightened for mounting values of activation energy parameter. Few recent investigations explore the characteristics of activation energy for various fluids [25-29]. In many practical applications the stretching sheet may not be linear, exponential because it can be stretched radially also in this context axisymmetric flow of fluids towards a radially stretched surface has got special interest due to its industrial applications like polymer extrusion, glass blowing etc. Ariel [30] examined second grade fluid due to radially stretching surface and further he obtained numerical and analytical solutions. Later slip flow of a fluid over a radially stretching sheet was studied by Ariel [31]. Investigations revealed that flow and heat transfer analysis of liquids [32-36] towards a radially stretching surface under various thermo-physical aspects. Khan et al. [37] reported Cross fluid past a radially stretching disk and they observed that velocity field exhibited the decreasing nature with the effect the local Weissenberg number. Most of the erstwhile studies reveal that flow geometries are confined to linear, nonlinear, exponential. Therefore, prime intention of this current study is to examine the aspects of activation energy and chemical reaction on MHD flow of cross nanoliquid over a radially stretching surface under simultaneous effects of viscous dissipation with joule heating, slip, convective condition and radiation. In addition to this zero normal flux condition is imposed at the sheet to spread the particles away from the sheet. Further detailed graphical analysis has been deliberated.

\section{FORMULATION}

Consider a steady Darcy Forchheimer flow of Cross nanoliquid past a radially stretching surface placed at $z=0$ and fluid resides in the region $z \geq 0$ with velocity $u=U w=$ ar, where a is positive number (see Figure 1). A uniform magnetic field $\mathrm{B}_{0}$ imposed normal to the disk. The heat transport mechanism is presented in presence of convective heating with temperature $T_{f}$ which gives a heat transfer coefficient $h_{f}$. Here $\mathrm{T}$ is the temperature of the fluid, $T_{\infty}$ is the temperature far away from the disk. Furthermore, effects of viscous dissipation, joule heating and radiation are assumed in heat transfer. Impacts of $\mathrm{AE}$ and $\mathrm{BCR}$ are taken into consideration in mass transfer.

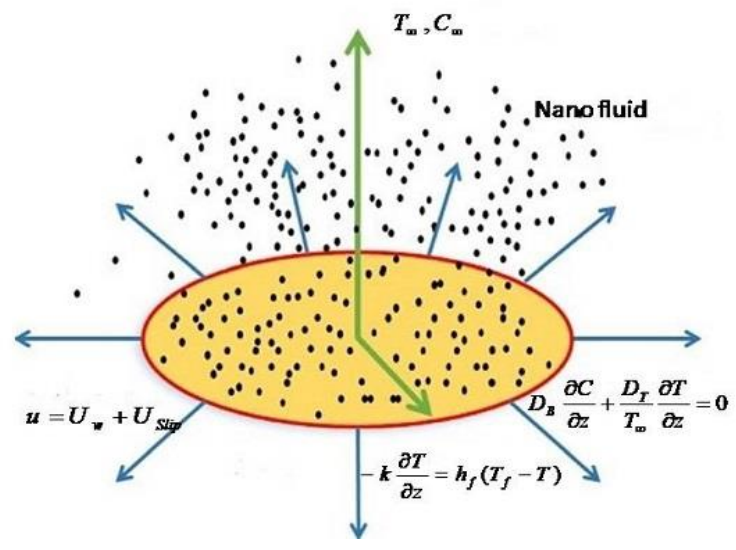

Figure 1. Geometry of the problem

By employing aforementioned aspects, the fundamental equations of mass, momentum, energy as well as concentration for Darcy Forchheimer flow of Cross nanoliquid with $\mathrm{AE}$ and $\mathrm{BCR}$ are modeled as:

$$
\begin{gathered}
\frac{\partial u}{\partial r}+\frac{u}{r}+\frac{\partial w}{\partial z}=0 \\
u \frac{\partial u}{\partial r}+w \frac{\partial u}{\partial z}=v \frac{\partial}{\partial z}\left(\frac{\frac{\partial u}{\partial z}}{1+\left(\Gamma \frac{\partial u}{\partial z}\right)^{n}}\right)-\frac{\sigma B_{0}^{2}}{\rho} u-\frac{v}{K} u \\
-\frac{C_{b}}{r \sqrt{K}} u^{2} \\
u \frac{\partial T}{\partial r}+w \frac{\partial T}{\partial z}=\alpha \frac{\partial^{2} T}{\partial z^{2}}+\tau\left[D_{B} \frac{\partial C}{\partial z} \frac{\partial T}{\partial z}+\frac{D_{T}}{T_{\infty}}\left(\frac{\partial T}{\partial z}\right)^{2}\right] \\
-\frac{1}{\rho c_{p}} \frac{\partial q_{r}}{\partial z}+\frac{\mu_{0}}{\rho C_{p}}\left(\frac{\left.\frac{\partial u}{\partial z}\right)^{2}}{1+\left(\Gamma \frac{\partial u}{\partial z}\right)^{n}}\right)^{2} \\
+\frac{\sigma B_{0}^{2}}{\rho} u^{2}
\end{gathered}
$$




$$
\begin{aligned}
u \frac{\partial C}{\partial r}+w \frac{\partial C}{\partial z}=D_{B} & \frac{\partial^{2} C}{\partial z^{2}}+\frac{D_{T}}{T_{\infty}} \frac{\partial^{2} T}{\partial z^{2}} \\
& -K_{r}^{2}\left(\frac{T}{T_{\infty}}\right)^{n} \exp \left(\frac{-E_{a}}{\kappa T}\right)\left(C-C_{\infty}\right)
\end{aligned}
$$

With allied boundary conditions,

$$
\left.\begin{array}{c}
u=u_{w}(r)+u_{\text {slip }}, \\
w=0,-k \frac{\partial T}{\partial Z}=h_{f}\left(T_{f}-T\right), \\
D_{B} \frac{\partial C}{\partial z}+\frac{D_{T}}{T_{\infty}} \frac{\partial T}{\partial z}=0 \text { atz }=0, \\
u=0, T=T_{\infty}, C=C_{\infty} \text { as } \rightarrow \infty
\end{array}\right\}
$$

Here velocity slip is $U_{\text {slip }}=l \frac{\frac{\partial u}{\partial z}}{1+\left(\Gamma \frac{\partial u}{\partial z}\right)^{n}}$.

Here $(u, w)$ signifies the velocity in radial- $r$ and axial-z directions respectively, $F=\frac{C_{b}}{r \sqrt{K}}, \alpha$ is thermal diffusivity $\sigma$ is electrical conductivity, $\mathrm{v}$ is the kinematic viscosity, $\rho$ is the density of the base fluid, $\mathrm{D}_{\mathrm{B}}$ is the Brownian diffusion coefficient an $\mathrm{D}_{\mathrm{T}}$ is the thermophoresis diffusion coefficient, $\tau=\frac{(\rho c)_{p}}{(\rho c)_{f}}$ is the ratio of nanoparticle heat capacity to the base fluid heat capacity. Using Rosseland approximation for radiation can write $q_{r}=-\frac{4 \sigma^{*}}{3 k^{*}} \frac{\partial T^{4}}{\partial z}$ with $\sigma^{*}$ is StefanBoltzmann parameter and $\mathrm{k}^{*}$ is denoted for mean absorption coefficient. Expansion of Taylor's series about the origin $T_{\infty}$ and by ignoring the highest order expressions we get $q_{r}=$ $-\frac{16 \sigma^{*} T_{\infty}^{3}}{3 k^{*}} \frac{\partial T}{\partial z}$ using this Eq. (3) takes the following form.

$$
\begin{aligned}
u \frac{\partial T}{\partial r}+w \frac{\partial T}{\partial z}= & \alpha \frac{\partial^{2} T}{\partial z^{2}}+\tau\left[D_{B} \frac{\partial C}{\partial z} \frac{\partial T}{\partial z}+\frac{D_{T}}{T_{\infty}}\left(\frac{\partial T}{\partial z}\right)^{2}\right] \\
& +\frac{16 \sigma^{*} T_{\infty}^{3}}{3 k^{*} \rho c_{p}} \frac{\partial^{2} T}{\partial z^{2}} \\
& +\frac{\mu_{0}}{\rho C_{p}}\left(\frac{\left(\frac{\partial u}{\partial z}\right)^{2}}{1+\left(\Gamma \frac{\partial u}{\partial z}\right)^{n}}\right)+\frac{\sigma B_{0}^{2}}{\rho} u^{2}
\end{aligned}
$$

Introducing the similarity variables,

$$
\left.\begin{array}{c}
\eta=\sqrt{\frac{a}{v} z, u=\operatorname{arf}^{\prime}(\eta), w=-2 \sqrt{a v} f(\eta),} \\
\theta=\frac{T-T_{\infty}}{T_{f}-T_{\infty}}, \phi=\frac{C-C_{\infty}}{C_{\infty}}
\end{array}\right\}
$$

By using Eq. (7), equation of continuity is trivially satisfied while Eqns. (2-4) and (6) are transformed into ODE form.

$$
\begin{aligned}
{[1+(1-n)(W e} & \left.\left.f^{\prime \prime}\right)^{n}\right] f^{\prime \prime \prime} \\
+ & {\left[2 f f^{\prime \prime}-(1+F r) f^{\prime 2}-(M\right.} \\
+ & \left.D a) f^{\prime}\right]\left\{1+\left(W e f^{\prime \prime}\right)^{n}\right\}^{2}=0 \\
(1+R d) \theta^{\prime \prime}+ & P r \\
& \left(2 f \theta^{\prime}+N b \theta^{\prime} \phi^{\prime}+N t \theta^{\prime 2}\right) \\
& +\operatorname{Pr}\left[M E c f^{\prime 2}\right. \\
& \left.+E c \frac{f^{\prime \prime}}{1+\left(W e f^{\prime \prime}\right)^{n}}\right]=0
\end{aligned}
$$

$$
\begin{aligned}
\phi^{\prime \prime}+2 S c f \phi^{\prime}+\frac{N t}{N b} & \theta^{\prime \prime} \\
& -S c \beta\left(1+\delta_{1} \theta\right)^{n} \exp \left(\frac{-E}{1+\delta_{1} \theta}\right) \phi \\
& =0
\end{aligned}
$$

Corresponding transformed boundary conditions are:

$$
\begin{gathered}
f=0, f^{\prime}=1+\delta f^{\prime \prime}(0)\left\{\frac{1}{1+\left(W e f^{\prime \prime}(0)\right)^{n}}\right\}, \\
\theta^{\prime}=-\gamma[1-\theta(0)], N b \phi^{\prime}+N t \theta^{\prime}=0 \text { at } \eta=0, \\
f^{\prime} \rightarrow 0, \theta \rightarrow 0, \phi \rightarrow 0 \text { as } \eta \rightarrow \infty
\end{gathered}
$$

The physical parameters appeared in above equations are Weissenberg number $W e=\Gamma a R e_{r}^{1 / 2}$, inertia coefficient $F r=$ $\frac{C_{b}}{\sqrt{K}}$, porosity parameter $D a=\frac{v}{K a}$, magnetic parameter $M=\frac{\sigma B_{0}^{2}}{\rho a}$, Radiation parameter $R d=\frac{16 \sigma^{*} T_{\infty}^{3}}{3 k^{*} k_{1}}$, Prandtl number $\operatorname{Pr}=\frac{v}{\alpha}$, Eckert number $E c=\frac{u_{W}^{2}}{C_{p}\left(T_{f}-T_{\infty}\right)}$, Brownian motion parameter $N b=\frac{\tau D_{B} C_{\infty}}{v}$, thermophoresis parameter $N t=\frac{D_{T}\left(T_{f}-T_{\infty}\right)}{T_{\infty} v}$, energy parameter $E=\frac{E_{a}}{\kappa T_{\infty}}$, chemical reaction parameter $\beta=$ $\frac{K_{r}^{2}}{a}$, temperature difference $\delta=\frac{\left(T_{f}-T_{\infty}\right)}{T_{\infty}}$, Schmidt number $S c=$ $\frac{v}{D_{B}}$, Biot number $\gamma=\sqrt{\frac{v}{a}} \frac{h_{f}}{k}$.

Skin friction coefficient $C f_{r}$ and the local Nusselt number $N u_{r}$ are:

$$
\begin{aligned}
& C f_{r}=\frac{\left(\tau_{r z}\right)_{z=0}}{\frac{1}{2} \rho u_{w}^{2}}, N u_{r}=\frac{r q_{w}}{k\left(T_{f}-T_{\infty}\right)} \text { where }\left(\tau_{r z}\right)_{z=0}= \\
& {\left[\eta_{0} \frac{\frac{\partial u}{\partial z}}{1+\left(\Gamma \frac{\partial u}{\partial z}\right)^{n}}\right]_{z=0}, q_{w}=-k\left(\frac{\partial T}{\partial z}\right)_{z=0} .} \\
& \frac{1}{2} R e_{r}^{1 / 2} C f_{r}=\frac{f^{\prime \prime}(0)}{1+\left(W e f^{\prime \prime}(0)\right)^{n}}, R e_{r}^{-1 / 2} N u_{r}=-\theta^{\prime}(0) \text { where } \\
& R e_{r}=\frac{u_{w}(r) r}{v} .
\end{aligned}
$$

\section{METHODOLOGY}

Nonlinear system (8-10) subject to (11) is computed numerically by Runge-Kutta-Fehlberg method cum shooting technique. To verify the present numerical outcomes, we have compared our numerical results of $-\theta^{\prime}(0)$ with Butt and Ali [38] in Table 1.

Table 1. Comparison results of $-\theta^{\prime}(0)$ for Newtonian case $W e=0$ and $F r=0, D a=0$ and $P r=1$

\begin{tabular}{ccccc}
\hline$M$ & $\gamma$ & $E c$ & Butt and Ali [38] & Present results \\
\hline 0 & 0.5 & 0.5 & 0.21914 & 0.21913627 \\
0.5 & & & 0.16621 & 0.16620886 \\
1.0 & & & 0.11776 & 0.11776369 \\
2.0 & & & 0.02992 & 0.02992232 \\
1.0 & 0.1 & & 0.03425 & 0.03424722 \\
& 0.2 & & 0.06151 & 0.06151184 \\
& 0.5 & & 0.11776 & 0.11776369 \\
& 1.0 & & 0.16940 & 0.16940257 \\
\hline
\end{tabular}

\section{RESULTS AND DISCUSSION}

This segment is presented to analyze the physical 
implication of various fluid parameters $M$, Weissenberg number $(W e)$, porosity parameter $(D a)$, inertia coefficient $(F r)$, Prandtl number $(P r)$, Eckert number $(E c)$, thermophoresis parameter $(N t)$, Brownian motion parameter $(N b)$, Schmidt number $(S c)$, chemical reaction parameter $(\beta)$, energy $(E)$, Biot number $(\gamma)$, slip parameter $(\delta)$ on velocity $f^{\prime}(\eta)$, temperature $\theta(\eta)$ and concentration fields $\phi(\eta)$. Table 1 is prepared to compare the present results with the existing literature. Figure 2 captured to examine the impact of $M$ on $f^{\prime}(\eta)$ and it elucidate that larger estimation of $M$ decays the velocity field. Physically mounted values of $M$ mean Lorentz force generates more resistance to the fluid therefore fluid velocity depreciates and reduces momentum boundary layer thickness. Effect of $M$ on $\theta(\eta)$ is visualized through Figure 3 . It is clear from the figure that $\theta(\eta)$ is enhanced with the stronger magnetic field. As the Lorentz force is resistive force which restricts the fluid motion. Therefore, more heat is produced and thus temperature raised in the working fluid. From the Figure 4 it is clear that $\phi(\eta)$ raises with the enlargement of $M$. Figure 5 demonstrates the characteristics of Weissenberg number We on velocity $f^{\prime}(\eta)$. It is seen from the figure velocity deteriorates by uplifting the values of We for shear thinning fluid. Further it is noticeable from the Figure 6 and Figure 7 that cross nanoliquid temperature and concentration enhances for greater We. Physically, enlarging the values of the We causes enhancement in the relaxation time which results dwindle the velocity and rise in temperature. Figures 8-10 are plotted to examine the influence of $\delta$ on $f^{\prime}(\eta), \theta(\eta)$ and $\phi(\eta)$. Velocity profile exhibits decreasing trend for greater values of slip parameter while opposite tendency is noticed for $\theta(\eta)$ and $\phi(\eta)$. Figures 11-13 illustrates the influence of porosity parameter on $f^{\prime}(\eta), \theta(\eta)$ and $\phi(\eta)$. It has been observed that greater porosity parameter leads to lower velocity, higher temperature and concentration for the fluid.

Physically the existence of porous space enhances the resistance to liquid flow which results in increase in temperature and concentration. Figure 14 exhibits the behavior of velocity profile $f^{\prime}(\eta)$ due to inertia coefficient $F r$. It is evident that velocity declined for upsurging $F r$ and corresponding momentum layer gets thicker. Variation of velocity temperature and concentration field through powerlaw index $n$ is examined in Figures 15-17. Figure 15 reveals that $f^{\prime}(\eta)$ and momentum layer exhibits a progressive tendency for greater values of $n$ but temperature and concentration field declined corresponding to uprising $n$ (Figure 16 and Figure 17). In physical point of view shearthinning liquid experiences low resistive force due to the low viscosity which causes for increment in the $f^{\prime}(\eta)$ and declining of $\theta(\eta)$. Figure 18 reports the variation of $\theta(\eta)$ due to $\gamma$. An enlargement in $\gamma$ causes stronger convection, consequently temperature increment is noticed.

Attribute of $E c$ displayed in Figure 19. Here greater $E c$ yields higher $\theta(\eta)$. Larger values of $E c$ causes to increase resistance in fluid motion as a consequence of this much heat is produced hence temperature profile increases. Influence of Nt on $\theta(\eta)$ and $\phi(\eta)$ is disclosed via Figure 20 and Figure 21. Clearly a rise in $N t$ augments temperature and concentration. Physically, thermophoresis force increases with enhancement of $N t$ due to these nanoparticles are being pushed towards cold zone from hot zone and hence increase in the $N t$ temperature and $\phi(\eta)$ increases. Figure 22 is sketched to discuss the variation of $\phi(\eta)$ due to $N b$. It is detected that $\phi(\eta)$ exhibited decreasing tendency for greater values of $\mathrm{Nb}$. It is happened due the uneven movement of nanoparticles in the fluid. Figure 23 interprets the role of $R d$ on $\theta(\eta)$. From the figure it is described that larger values of radiation parameter enhance the temperature profile $\theta(\eta)$. Physically radiation process generates much heat in the liquid and corresponding thermal layer gets thicker.

Influence of $E$ on $\phi(\eta)$ is discussed through Figure 24. It is noticed that concentration is increasing function of $E$. Physically, uplifting the values of $E$ reduce the Arrhenius energy function which grows the generative chemical reaction, consequently $\phi(\eta)$ augmented. Figure 25 depicts the effects of chemical reaction rate $\beta$ on $\phi(\eta)$. We observed that if we upgrade $\beta$ a plummeting nature is observed in $\phi(\eta)$. Table 2 and Table 3 represents the variation in Skin friction coefficient $C f_{r}$ and the local Nusselt number $N u_{r}$ for various parameters.

Table 2. Numerical values of $C f_{r} R e_{r}^{\frac{1}{2}}$ for various parameters

\begin{tabular}{ccccccc}
\hline $\mathbf{M}$ & $\mathbf{n}$ & $\mathbf{W e}$ & $\mathbf{F r}$ & $\mathbf{D a}$ & $\boldsymbol{\delta}$ & $\boldsymbol{C}_{\boldsymbol{f r}} \boldsymbol{R} \boldsymbol{e}_{\boldsymbol{r}}^{\frac{1}{2}}$ \\
\hline 0.0 & 0.5 & 0.8 & 0.5 & 0.5 & 0.2 & 0.86428297 \\
0.4 & & & & & & 0.92602313 \\
0.8 & & & & & & 0.98085549 \\
0.4 & 0.2 & & & & & 0.94573346 \\
& 0.4 & & & & & 0.93358237 \\
& 0.6 & & & & & 0.91720084 \\
& & 0.1 & & & & 1.20373753 \\
& 0.2 & & & & 1.17220248 \\
& 0.3 & & & & 1.13065507 \\
& & 0.3 & & & 0.91052507 \\
& & & 0.6 & & & 0.93356439 \\
& & & & 0.1 & & 0.95541627 \\
& & & & 0.3 & & 0.86428297 \\
& & & & 0.5 & & 0.92602313 \\
& & & & & 0.1 & 1.03252077 \\
& & & & & 0.3 & 0.92602313 \\
& & & & & & 0.84031436 \\
\hline
\end{tabular}

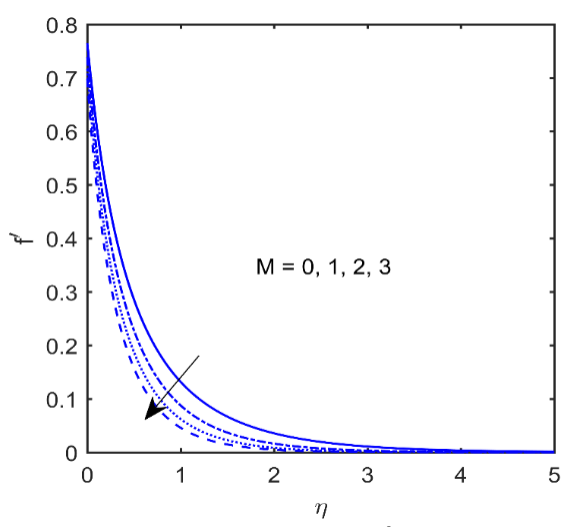

Figure 2. Variations of $f^{\prime}(\eta)$ via $M$

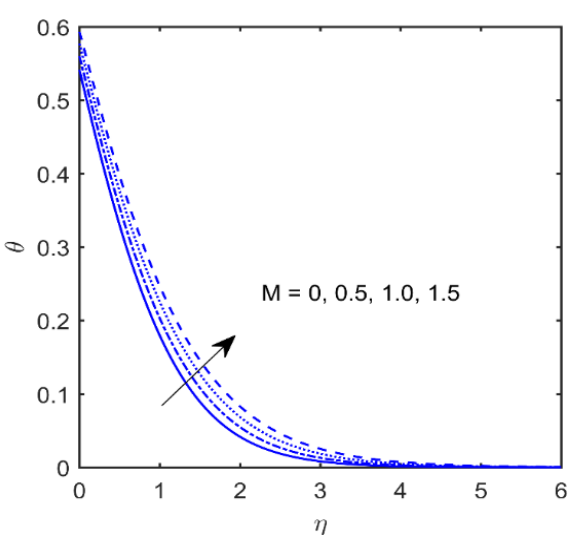

Figure 3. Variations of $\theta$ via $M$ 


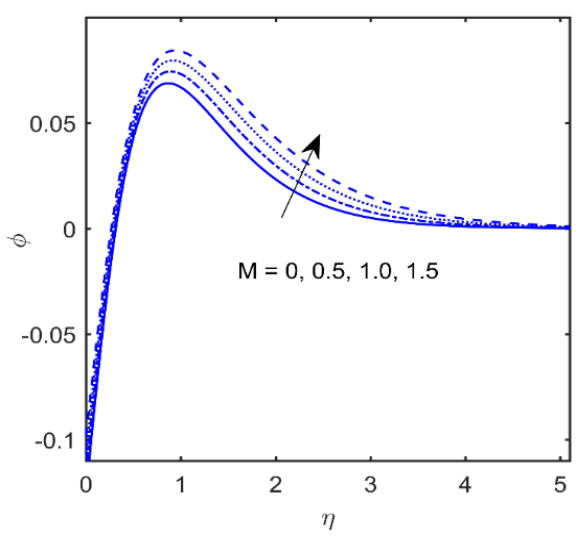

Figure 4. Variations of $\phi$ via $M$

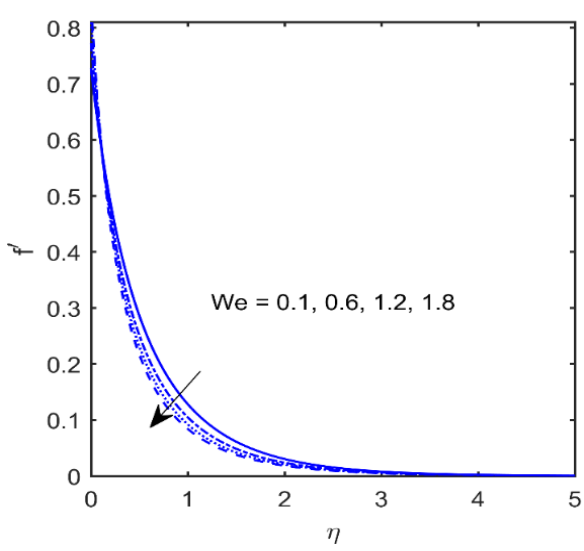

Figure 5. Variations of $f^{\prime}(\eta)$ via $W e$

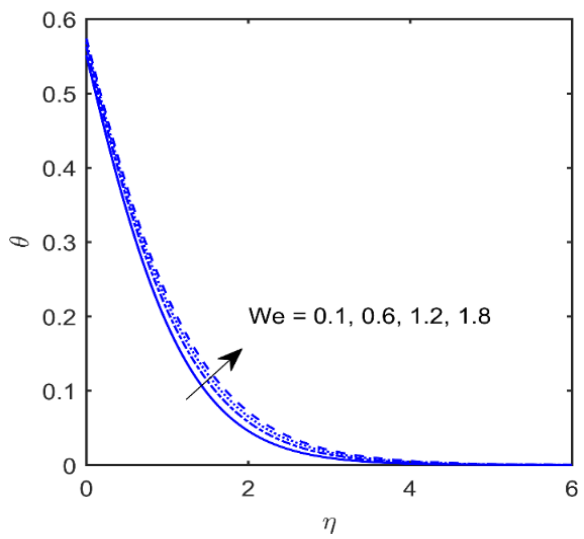

Figure 6. Variations of $\theta$ via $W e$

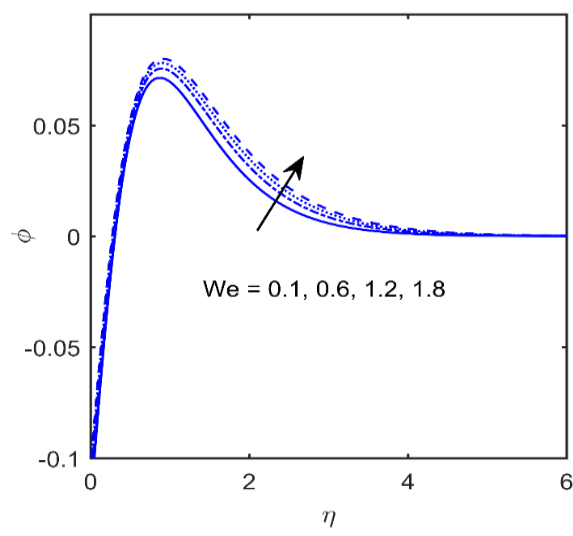

Figure 7. Variations of $\phi$ via $W e$

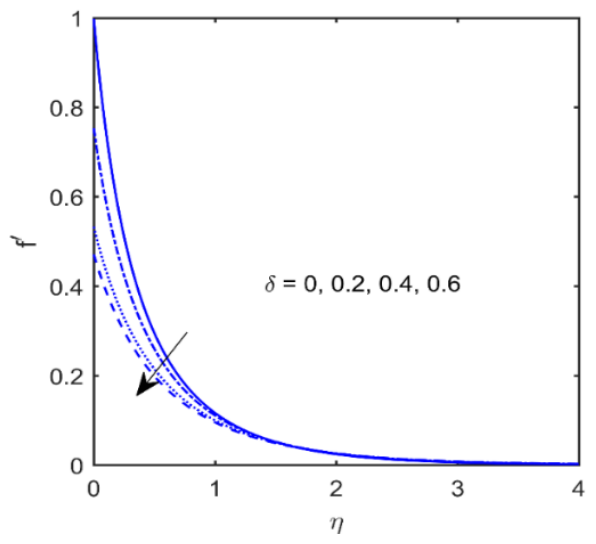

Figure 8. Variations of $f^{\prime}(\eta)$ via $\delta$

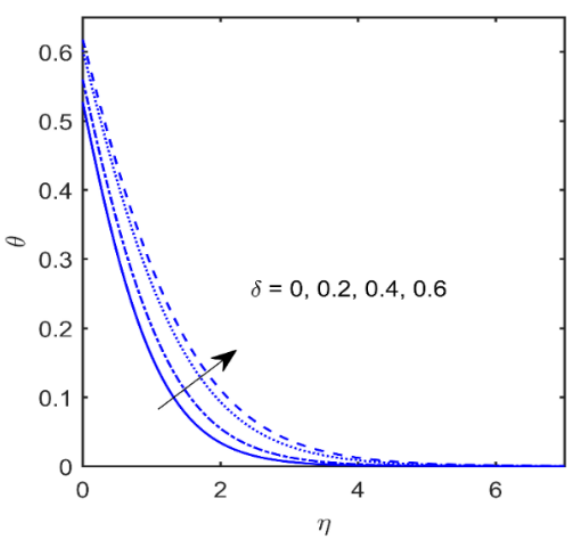

Figure 9. Variations of $\theta$ via $\delta$

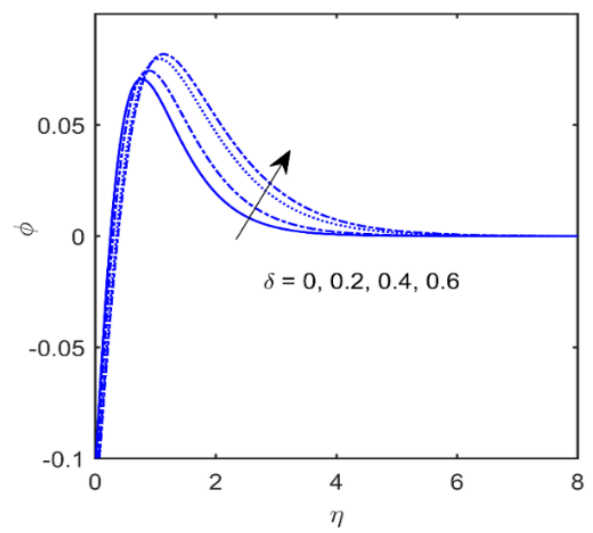

Figure 10. Variations of $\phi$ via $\delta$

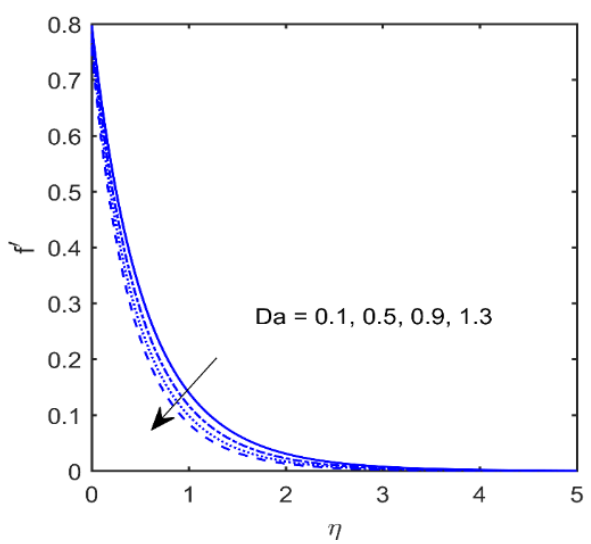

Figure 11. Variations of $f^{\prime}(\eta)$ via $D a$ 


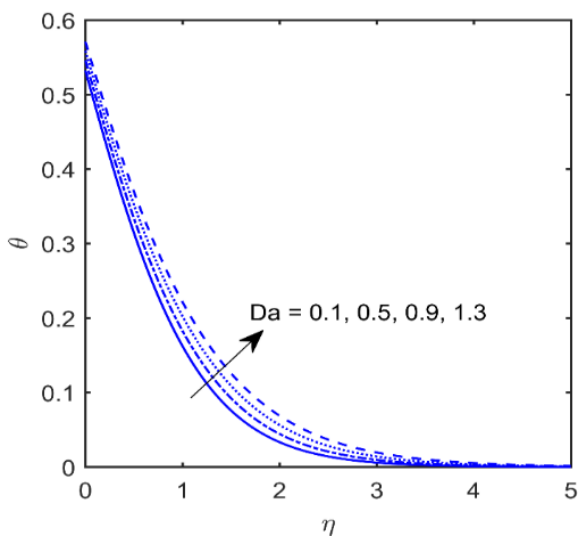

Figure 12. Variations of $\theta$ via $D a$

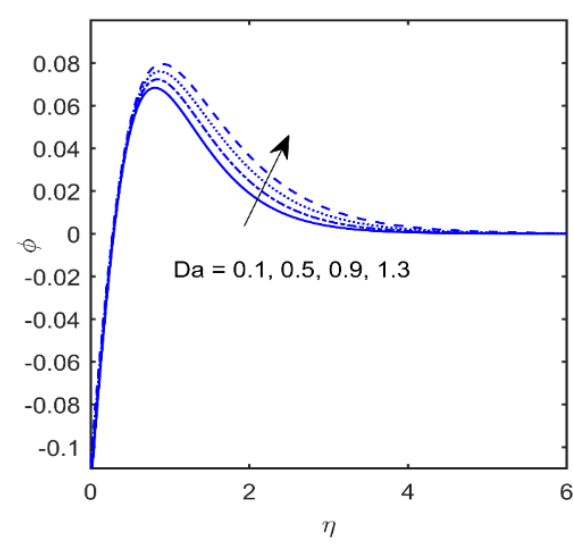

Figure 13. Variations of $\phi$ via $D a$

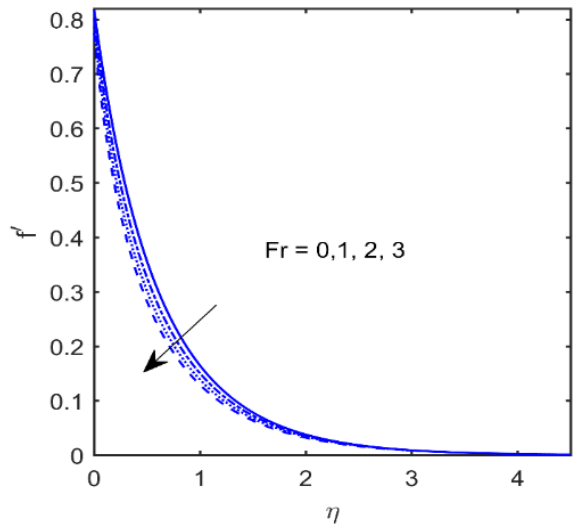

Figure 14. Variations of $f^{\prime}(\eta)$ via $F r$

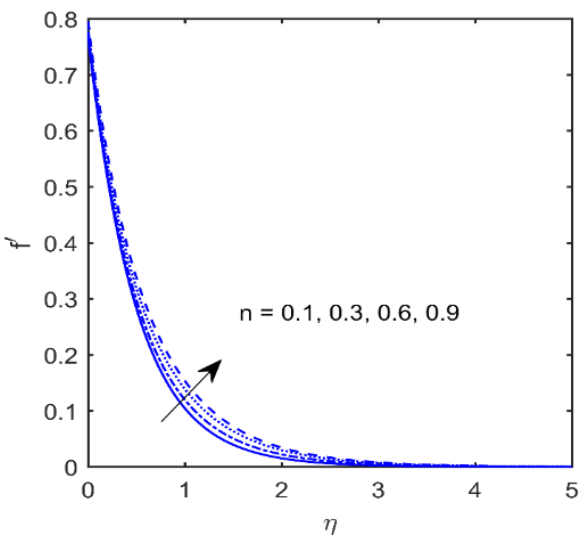

Figure 15. Variations of $f^{\prime}(\eta)$ via $n$

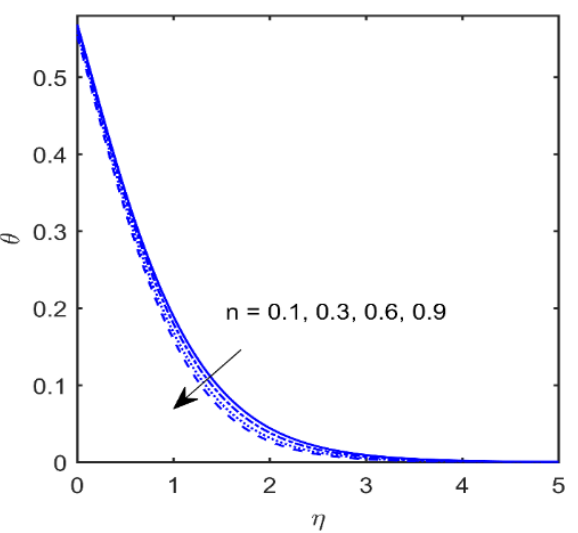

Figure 16. Variations of $\theta$ via $\mathrm{n}$

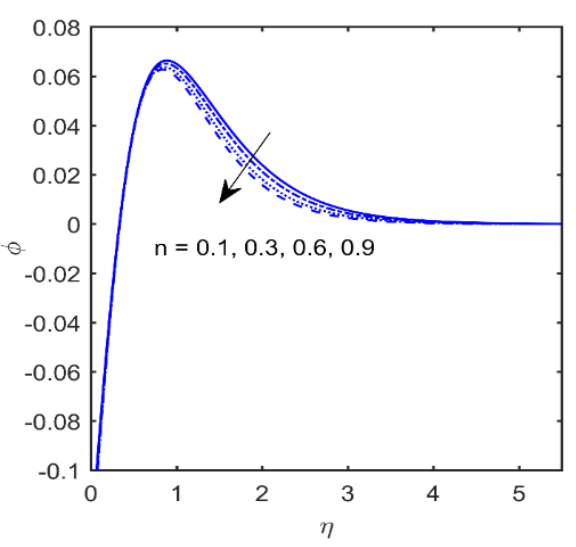

Figure 17. Variations of $\phi$ via $n$

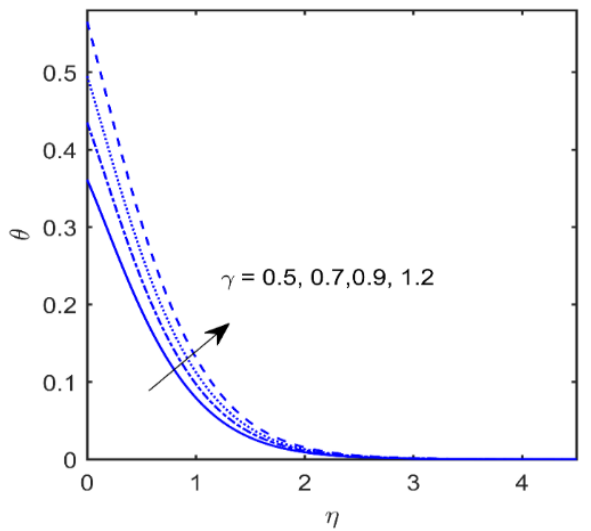

Figure 18. Variations of $\theta$ via $\gamma$

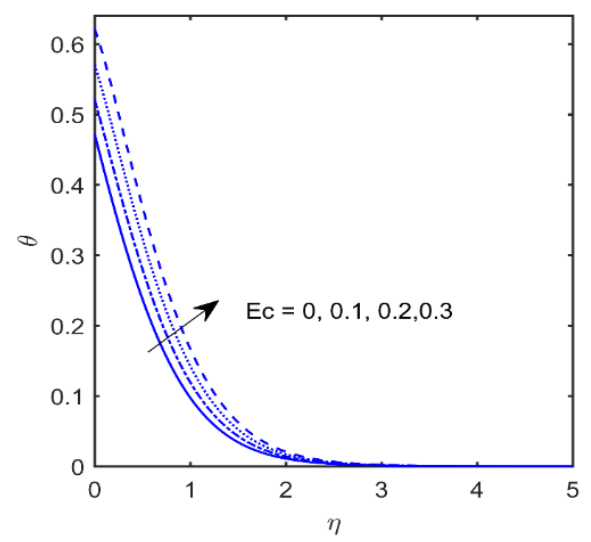

Figure 19. Variations of $\theta$ via $E c$ 


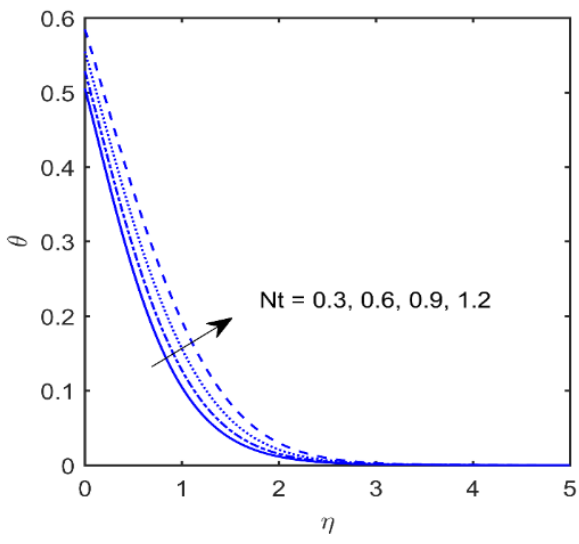

Figure 20. Variations of $\theta$ via $\mathrm{Nt}$

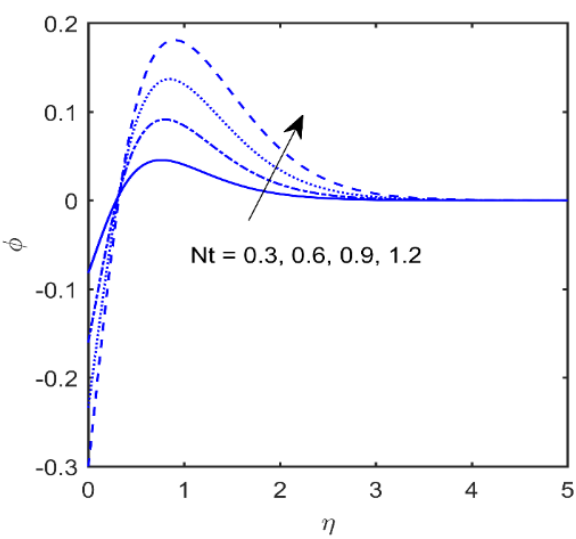

Figure 21. Variations of $\phi$ via $N t$

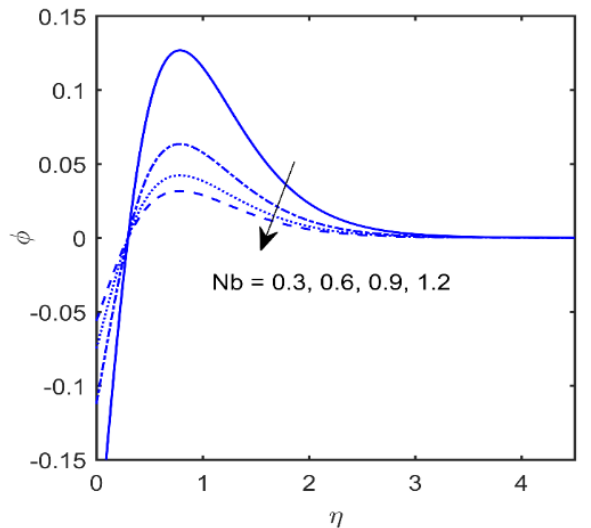

Figure 22. Variations of $\phi$ via $N b$

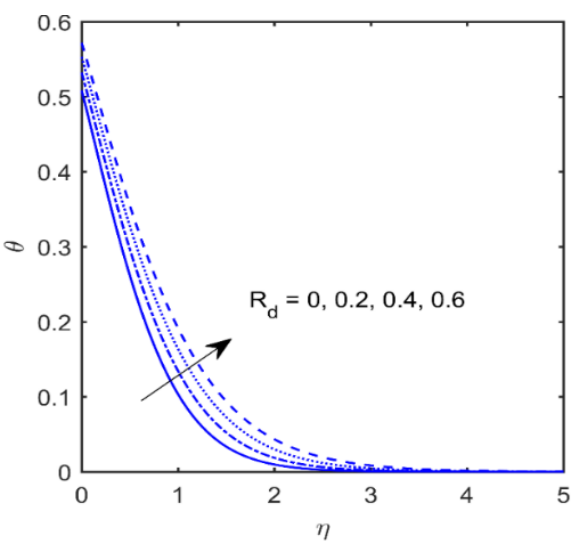

Figure 23. Variations of $\theta$ via $R d$

Table 3. Numerical values of $N u_{r} R e_{r}^{-\frac{1}{2}}$ for various parameters

\begin{tabular}{|c|c|c|c|c|c|c|c|c|c|c|}
\hline $\mathbf{M}$ & n & We & $\mathbf{R d}$ & Ec & $\mathbf{N t}$ & Nb & $\mathbf{E}$ & B & $\gamma$ & $N u_{r} R e_{r}^{-\frac{1}{2}}$ \\
\hline 0.0 & 0.5 & 0.8 & 0.25 & 0.1 & 0.3 & 0.3 & 1 & 1 & 0.6 & 0.40817062 \\
\hline 0.4 & & & & & & & & & & 0.38939603 \\
\hline 0.8 & & & & & & & & & & 0.37210942 \\
\hline & 0.2 & & & & & & & & & 0.39024987 \\
\hline & 0.4 & & & & & & & & & 0.38973814 \\
\hline & 0.6 & & & & & & & & & 0.38897951 \\
\hline & & 0.1 & & & & & & & & 0.40122524 \\
\hline & & 0.2 & & & & & & & & 0.39821936 \\
\hline & & 0.3 & & & & & & & & 0.39610769 \\
\hline & & & 0.15 & & & & & & & 0.36689399 \\
\hline & & & 0.25 & & & & & & & 0.38939603 \\
\hline & & & 0.35 & & & & & & & 0.41094587 \\
\hline & & & & 0.0 & & & & & & 0.42448060 \\
\hline & & & & 0.1 & & & & & & 0.38939603 \\
\hline & & & & 0.2 & & & & & & 0.35401764 \\
\hline & & & & & 0.2 & & & & & 0.39368161 \\
\hline & & & & & 0.4 & & & & & 0.38497444 \\
\hline & & & & & 0.6 & & & & & 0.37570419 \\
\hline & & & & & & 0.2 & & & & 0.38939603 \\
\hline & & & & & & 0.4 & & & & 0.38939603 \\
\hline & & & & & & 0.6 & & & & 0.38939603 \\
\hline & & & & & & & 1 & & & 0.38939603 \\
\hline & & & & & & & 2 & & & 0.38990527 \\
\hline & & & & & & & 3 & & & 0.39003032 \\
\hline & & & & & & & & 1.5 & & 0.38889180 \\
\hline & & & & & & & & 2.5 & & 0.38799035 \\
\hline & & & & & & & & 3.5 & & 0.38726308 \\
\hline & & & & & & & & & 0.3 & 0.25066044 \\
\hline & & & & & & & & & 0.4 & 0.30522449 \\
\hline & & & & & & & & & 0.5 & 0.35081462 \\
\hline
\end{tabular}




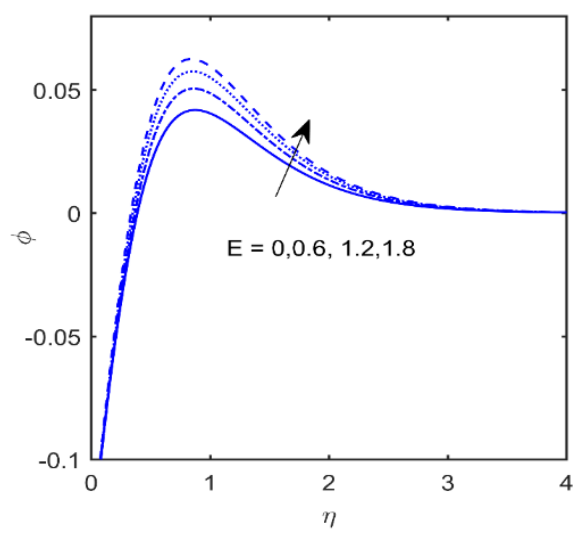

Figure 24. Variations of $\phi$ via $E$

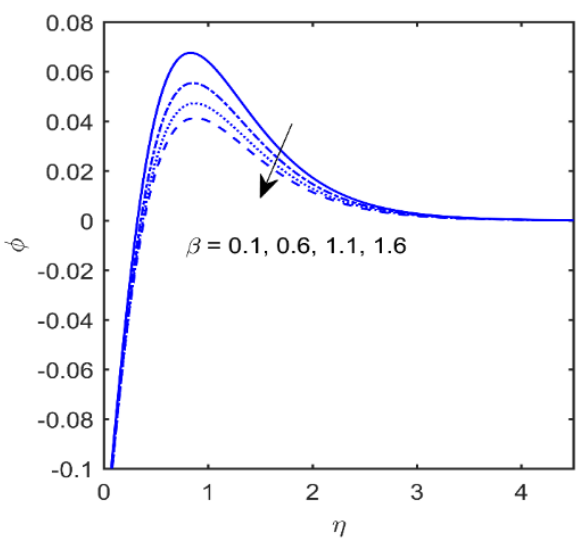

Figure 25. Variations of $\phi$ via $\beta$

\section{REFERENCES}

[1] Cross, M.M. (1965). Rheology of non-Newtonian fluids: A new flow equation for pseudoplastic systems. Journal of Colloid Science, 20(5): 417-437. https://doi.org/10.1016/0095-8522(65)90022-X

[2] Escudier, M.P., Gouldson, I.W., Pereira, A.S., Pinho, F.T., Poole, R.J. (2001). On the reproducibility of the rheology of shear-thinning liquids. Journal of NonNewtonian Fluid Mechanics, 97(2-3): 99-124. https://doi.org/10.1016/S0377-0257(00)00178-6

[3] Xie, J., Jin, Y.C. (2016). Parameter determination for the cross rheology equation and its application to modeling non-Newtonian flows using the WC-MPS method. Engineering Applications of Computational Fluid Mechanics, 10(1): 111-129. https://doi.org/10.1080/19942060.2015.1104267

[4] Khan, M., Manzur, M., Rahman, M.U. (2016). Boundary layer flow and heat transfer of cross fluid over a stretching sheet. Thermal Science, 23(1). http://dx.doi.org/10.2298/TSCI160919111K

[5] Forchheimer, P. (1901). Wasserbewegung durch Boden. 45th Edition, Zeitschrift des Vereins deutscher Ingenieure, Düsseldorf.

[6] Muskat, M. (1938). The flow of homogeneous fluids through porous media. International Human Resources Development, Williams \& Wilkins 1938, 46(2): 169.

[7] Seddeek, M.A. (2006). Influence of viscous dissipation and thermophoresis on Darcy-Forchheimer mixed convection in a fluid saturated porous media. Journal of Colloid and Interface Science, 293(1): 137-142. https://doi.org/10.1016/j.jcis.2005.06.039

[8] Pal, D., Mondal, H. (2012). Hydromagnetic convective diffusion of species in Darcy-Forchheimer porous medium with non-uniform heat source/sink and variable viscosity. International Communications in Heat and Mass Transfer, 39(7): 913-917. https://doi.org/10.1016/j.icheatmasstransfer.2012.05.01 2

[9] Shehzad, S.A., Abbasi, F.M., Hayat, T., Alsaedi, A. (2016). Cattaneo-Christov heat flux model for DarcyForchheimer flow of an Oldroyd-B fluid with variable conductivity and non-linear convection. Journal of Molecular Liquids, 224: 274-278. https://doi.org/10.1016/j.molliq.2016.09.109

[10] Hayat, T., Muhammad, T., Al-Mezal, S., Liao, S.J. (2016). Darcy-Forchheimer flow with variable thermal conductivity and Cattaneo-Christov heat flux. International Journal of Numerical Methods for Heat \& Fluid Flow, 26(8): 2355-2369. https://doi.org/10.1108/HFF-08-2015-0333

[11] Choi, S.U., Eastman, J.A. (1995). Enhancing thermal conductivity of fluids with nanoparticles. Argonne National Lab., IL (United States).

[12] Masuda, H., Ebata, A., Teramae, K., Hishinuma, N., Ebata, Y. (1993). Alteration of thermal conductivity and viscosity of liquid by dispersing ultra-fine particles (dispersion of $\gamma-\mathrm{Al}_{2} \mathrm{O}_{3}, \quad \mathrm{SiO}_{2}$ and $\mathrm{TiO}_{2}$ ultra-fine particles). NETSU BUSSEI, 7(4): 227-233. https://www.sid.ir/en/journal/ViewPaper.aspx?ID=3129 89.

[13] Buongiorno, J. (2006). Convective transport in nanofluids. J. Heat Transfer, 128(3): 240-250. https://doi.org/10.1115/1.2150834

[14] Khan, W.A., Pop, I. (2010). Boundary-layer flow of a nanofluid past a stretching sheet. International Journal of Heat and Mass Transfer, 53(11-12): 2477-2483. https://doi.org/10.1016/j.ijheatmasstransfer.2010.01.032

[15] Makinde, O.D., Aziz, A. (2011). Boundary layer flow of a nanofluid past a stretching sheet with a convective boundary condition. International Journal of Thermal Sciences, 50(7): $1326-1332$. https://doi.org/10.1016/j.ijthermalsci.2011.02.019

[16] Kuznetsov, A.V., Nield, D.A. (2014). Natural convective boundary-layer flow of a nanofluid past a vertical plate: A revised model. International Journal of Thermal Sciences, $\quad 77$ : 126-129. https://doi.org/10.1016/j.ijthermalsci.2013.10.007

[17] Kumar, K.G., Ramesh, G.K., Gireesha, B.J., Gorla, R.S.R. (2018). Characteristics of Joule heating and viscous dissipation on three-dimensional flow of Oldroyd B nanofluid with thermal radiation. Alexandria Engineering Journal, 57(3): 2139-2149. https://doi.org/10.1016/j.aej.2017.06.006

[18] Prabhakar, B., Bandari, S., Reddy, C.S. (2019). A revised model to analyze MHD flow of maxwell nanofluid past a stretching sheet with nonlinear thermal radiation effect. International Journal of Fluid Mechanics Research, 46(2): 151-165. https://doi.org/10.1615/InterJFluidMechRes.201802103 7

[19] Hayat, T., Ullah, I., Waqas, M., Alsaedi, A. (2018). Flow of chemically reactive magneto Cross nanoliquid with temperature-dependent conductivity. Applied $\begin{array}{lll}\text { Nanoscience, } & \text { 8(6): }\end{array}$ 
https://doi.org/10.1007/s13204-018-0813-x

[20] Azam, M., Shakoor, A., Rasool, H.F., Khan, M. (2019). Numerical simulation for solar energy aspects on unsteady convective flow of MHD Cross nanofluid: A revised approach. International Journal of Heat and Mass Transfer, 131: 495-505. https://doi.org/10.1016/j.ijheatmasstransfer.2018.11.022

[21] Bestman, A.R. (1990). Natural convection boundary layer with suction and mass transfer in a porous medium. International Journal of Energy Research,14(4): 389-396. https://doi.org/10.1002/er.4440140403

[22] Makinde, O.D., Olanrewaju, P.O., Charles, W.M. (2011). Unsteady convection with chemical reaction and radiative heat transfer past a flat porous plate moving through a binary mixture. Afrika Matematika, 22(1): 6578. https://doi.org/10.1007/s13370-011-0008-z

[23] Alsaadi, F.E., Ullah, I., Hayat, T., Alsaadi, F.E. (2019). Entropy generation in nonlinear mixed convective flow of nanofluid in porous space influenced by Arrhenius activation energy and thermal radiation. Journal of Thermal Analysis and Calorimetry, 140: 799-809. https://doi.org/10.1007/s10973-019-08648-0

[24] Irfan, M., Khan, W.A., Khan, M., Gulzar, M.M. (2019). Influence of Arrhenius activation energy in chemically reactive radiative flow of 3D Carreau nanofluid with nonlinear mixed convection. Journal of Physics and Chemistry of Solids, 125: 141-152. https://doi.org/10.1016/j.jpcs.2018.10.016

[25] Dhlamini, M., Kameswaran, P.K., Sibanda, P., Motsa, S., Mondal, H. (2019). Activation energy and binary chemical reaction effects in mixed convective nanofluid flow with convective boundary conditions. Journal of Computational Design and Engineering, 6(2): 149-158. https://doi.org/10.1016/j.jcde.2018.07.002

[26] Hayat, T., Riaz, R., Aziz, A., Alsaedi, A. (2020). Influence of Arrhenius activation energy in MHD flow of third grade nanofluid over a nonlinear stretching surface with convective heat and mass conditions. Physica A: Statistical Mechanics and Its Applications, 549:

124006 https://doi.org/10.1016/j.physa.2019.124006

[27] Sultan, F., Khan, W.A., Ali, M., Shahzad, M., Sun, H., Irfan, M. (2019). Importance of entropy generation and infinite shear rate viscosity for non-Newtonian nanofluid. Journal of the Brazilian Society of Mechanical Sciences and Engineering, 41(10): 439. https://doi.org/10.1007/s40430-019-1950-1

[28] Ijaz, M., Yousaf, M., El Shafey, A.M. (2020). Arrhenius activation energy and Joule heating for Walter-B fluid with Cattaneo-Christov double-diffusion model. Journal of Thermal Analysis and Calorimetry, 1-12. https://doi.org/10.1007/s10973-020-09270-1

[29] Shah, Z., Kumam, P., Deebani, W. (2020). Radiative MHD Casson nanofluid flow with activation energy and chemical reaction over past nonlinearly stretching surface through Entropy generation. Scientific Reports, 10(1): 1-14. https://doi.org/10.1038/s41598-020-611259

[30] Ariel, P.D. (2001). Axisymmetric flow of a second grade fluid past a stretching sheet. International Journal of Engineering Science, 39(5): 529-553. https://doi.org/10.1016/S0020-7225(00)00052-5

[31] Ariel, P.D. (2007). Axisymmetric flow due to a stretching sheet with partial slip. Computers \&

Mathematics with Applications, 54(7-8): 1169-1183. https://doi.org/10.1016/j.camwa.2006.12.063

[32] Sahoo, B. (2010). Effects of partial slip on axisymmetric flow of an electrically conducting viscoelastic fluid past a stretching sheet. Open Physics, 8(3): 498-508. https://doi.org/10.2478/s11534-009-0105-X

[33] Shateyi, S., Makinde, O.D. (2013). Hydromagnetic stagnation-point flow towards a radially stretching convectively heated disk. Mathematical Problems in Engineering. https://doi.org/10.1155/2013/616947

[34] Khan, M., Munir, A., Shahzad, A., Shah, A. (2015). MHD flow and heat transfer of a viscous fluid over a radially stretching power-law sheet with suction/injection in a porous medium. Journal of Applied Mechanics and Technical Physics, 56(2): 231-240. https://doi.org/10.1134/S002189441502008X

[35] Khan, M., Malik, R., Munir, A., Shahzad, A. (2016). MHD flow and heat transfer of Sisko fluid over a radially stretching sheet with convective boundary conditions. Journal of the Brazilian Society of Mechanical Sciences and $\quad$ Engineering, 38(4): 1279-1289. https://doi.org/10.1007/s40430-015-0437-y

[36] Hayat, T., Waqas, M., Shehzad, S.A., Alsaedi, A. (2015). MHD stagnation point flow of Jeffrey fluid by a radially stretching surface with viscous dissipation and Joule heating. Journal of Hydrology and Hydromechanics, 63(4): 311-317. https://doi.org/10.1515/johh-2015-0038

[37] Khan, M., Manzur, M., Rahman, M. (2017). On axisymmetric flow and heat transfer of Cross fluid over a radially stretching sheet. Results in Physics, 7: 37673772. https://doi.org/10.1016/j.rinp.2017.08.039

[38] Butt, A.S., Ali, A. (2014). Entropy analysis of magnetohydrodynamic flow and heat transfer over a convectively heated radially stretching surface. Journal of the Taiwan Institute of Chemical Engineers, 45(4): 1197-1203. https://doi.org/10.1016/j.jtice.2014.02.019

\section{NOMENCLATURE}

$B_{0}$

$\mathrm{C}$

$C_{f}$

$C_{p}$

$\mathrm{Da}$

$D_{B}$

$D_{T}$

E

Ec

$f$

$\mathrm{Fr}$

$k$

M

$\mathrm{Nb}$

$N t$

$n$

$\operatorname{Pr}$

$R d$

$R e_{r}$

Sc

$T$

$u_{w}$

$W e$

$u, w$
Strength of magnetic field

Nanoparticle volume fraction

Skin friction coefficient

Specific heat at constant temperature

Porosity parameter

Brownian diffusion

Thermophoretic diffusion coefficient

Activation energy

Eckert number

Dimensionless stream function

Inertia coefficient

Thermal conductivity

Magnetic parameter

Brownian motion parameter

Thermophoretic parameter

Power law exponent

Prandtl number

Radiation parameter

Reynolds number

Schmidt number

Temperature of fluid

Velocity of the stretching sheet

Weissenberg number

Velocity components in $r$ and $z$ directions 
Time material constant

Chemical reaction parameter

Biot number

Similarity independent variable

Kinematic viscosity

Dimensionless nanoparticle volume

fraction

Stream function

Electrical conductivity

Density $\delta_{1}$

$(\rho c)_{f}$

$(\rho c)_{p}$

\section{Subscripts}

$\infty$

w
Slip parameter

Temperature difference parameter

Heat capacities of nanofluid

Effective heat capacity of the nanoparticle
Ambient condition

Conditions at the wall 